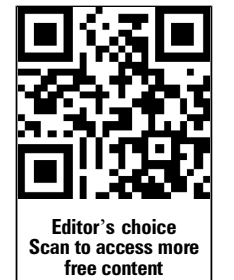

free content

${ }^{1}$ Schulthess Clinic, Zurich,

Switzerland

2Divisions of Emergency

Medicine and Sports Medicine, University of the

Witwatersrand, Johannesburg, South Africa

${ }^{3}$ Division of Cardiology,

Cardiovascular Centre

University Hospital Zurich,

Zurich, Switzerland

${ }^{4}$ Department of Family

Medicine, University of

Washington, Seattle,

Washington, USA

${ }^{5}$ Department of Anaesthetics,

Imperial College Healthcare

NHS Trust, London, UK

${ }^{6}$ Morningside Sports Medicine,

Johannesburg and the Section

of Sports Medicine, Faculty of

Health Sciences, University of

Pretoria, Pretoria, South Africa

${ }^{7}$ Local Organising Committee,

2014 FIFA Football World Cup

Brazil

${ }^{8}$ Department of Orthopedics,

University of São Paulo

Medical School, São Paulo,

Brazil

${ }^{9}$ Santa Monica Orthopaedic and Sports Medicine Group,

Santa Monica, California, USA

Correspondence to

Professor Jiri Dvorak,

Schulthess Clinic, Lengghalde 2,

Zurich 8008, Switzerland;

jiri.dvorak@f-marc.com

Received 17 June 2013

Accepted 25 June 2013

Published Online First

12 August 2013
To cite: Dvorak J,

Kramer EB, Schmied CM,

et al. Br I Sports Med

2013:47:1199-1202.

\title{
The FIFA medical emergency bag and FIFA 11 steps to prevent sudden cardiac death: setting a global standard and promoting consistent football field emergency care
}

\author{
Jiri Dvorak, ${ }^{1}$ Efraim B Kramer, ${ }^{2}$ Christian M Schmied, ${ }^{3}$ Jonathan A Drezner, ${ }^{4}$ \\ David Zideman, ${ }^{5}$ Jon Patricios, ${ }^{2,6}$ Luis Correia, ${ }^{7}$ André Pedrinelli, ${ }^{8}$ Bert Mandelbaum ${ }^{9}$
}

\begin{abstract}
Life-threatening medical emergencies are an infrequent but regular occurrence on the football field. Proper prevention strategies, emergency medical planning and timely access to emergency equipment are required to prevent catastrophic outcomes. In a continuing commitment to player safety during football, this paper presents the FIFA Medical Emergency Bag and FIFA 11 Steps to prevent sudden cardiac death. These recommendations are intended to create a global standard for emergency preparedness and the medical response to serious or catastrophic on-field injuries in football.
\end{abstract}

\section{INTRODUCTION}

Football is the most popular sport in the world. ${ }^{1}$ Medical emergencies on the football field are not common $^{2}$ but, if and when they occur, it is imperative that immediate recognition, on-field response and acute medical management are available to the player. Such emergency treatment is most efficiently administered by on-duty medical personnel ${ }^{3}$ using the appropriate equipment, ${ }^{4}$ until such time as emergency medical services arrive on scene to assist with additional treatment and/or transfer of the player $^{5}$ to the nearest, most appropriate medical facility. ${ }^{6}$

Sudden cardiac arrest (SCA) remains the leading cause of sudden death during football. The infrequent yet regular occurrence of SCA on the football field can to a large extent be prevented, and if necessary, effectively treated by immediate cardiopulmonary resuscitation (CPR) and automated external defibrillator (AED) use. $^{7-9}$

To support and promote a standardized ${ }^{10}$ and consistent level of advanced life support and emergency medical care on the football field, the FIFA Medical Assessment and Research Center (F-MARC) has developed a FIFA Medical Emergency Bag (FMEB) for distribution to all 209 member association (MA) medical departments. F-MARC also has developed the 'FIFA 11 Steps to prevent SCD' which outline fundamental measures to prevent sudden cardiac death (SCD) in football. The 11 steps are aimed at raising the level of awareness and appropriate action for all those involved with football internationally. These efforts follow the decision of the FIFA Congress 2012 (Budapest) to provide each of the 209 MAs with an AED as an indication of the importance of the prevention of sudden cardiac death. F-MARC further endorsed the Congress decision by creating a complete FMEB including educational manual and instructional video (http://www.F-MARC.com).

\section{Setting a standard}

The FMEB was developed by a process of expert consultation and consensus encompassing a group of football-experienced and actively involved medical specialists encompassing the fields of neurology, cardiology, orthopaedic surgery, sports and emergency medicine from countries including Switzerland, England, the USA, Brazil and South Africa.

The FMEB is intended for use by all FIFA MAs internationally, for training ${ }^{11}$ and competition. It is the recommended emergency medical bag for football team physicians, field-of-play medical teams and other medical professionals on duty during football events. Although it is acknowledged and appreciated that certain FIFA MAs may wish to provide a level of football emergency medical care above that provided by the contents of the FMEB, the FMEB takes into consideration the global nature of football. The FMEB provides a benchmark of optimal football emergency care that all MAs should strive for during training and competition, irrespective of the location.

Therefore, although individual or team medical personnel may use their own discretion to add items to the FMEB due to local circumstances, experience and personnel skills, it is recommended that the basic inventory of the FMEB should not be altered and always be available. This will ensure consistency of the core contents across venues and enable healthcare professionals from different teams to be able to work together, on any field-of-play emergency, combining response efforts and FMEBs with the same basic contents.

\section{FMEB content considerations}

The decision regarding appropriate contents of a 'universal' football emergency medical bag must take into consideration the multitude of locations globally where football is played, at amateur and professional levels. ${ }^{12}$ Every week millions of registered matches are played worldwide. Additionally, regard should be given to the varied emergency medical knowledge, training, skills and experience and the varied composition of healthcare professional teams who are on-duty at the football 
field-of-play during training or competition. In the absence of adequate competency in the recommended life-saving medical skills related to the contents of the FMEB or knowledge of the current standards of care of the expected football field emergency medical conditions, these inadequacies should be improved by implementation of the FIFA football emergency medicine training courses, undertaken locally by the confederations and/or MAs for team physicians, venue medical officers and field-of-play medical team members. ${ }^{13}$ Additional qualifications such as prehospital basic ${ }^{14}$ and advanced life support qualifications are strongly recommended as the minimum level of skill competency and knowledge.

\section{Contents of the FMEB}

In considering the contents of a 'universal' emergency medical bag, it was agreed that the level of medical care envisaged should be practically equivalent to an advanced life support level of emergency care. After setting the intended level of emergency care, consideration was centred on (i) which medical emergencies would likely be encountered on the football field (box 1) and (ii) which relevant basic medical equipment, encompassing at least 'Airway, Breathing and Circulation' considerations, an on-duty field-of-play healthcare professional could be expected to have competence in using.

It was also agreed that the contents of the FMEB (table 1) would primarily be designed to medically manage a player for approximately $60 \mathrm{~min}$ who was older than 14 years of age, with a weight of $50 \mathrm{~kg}$ or greater. The inclusion of a rigid, durable immobilisation-carrying device was considered mandatory. ${ }^{15}$ Although the item recommended was a toughened plastic-type long spinal board or equivalent to provide universal availability, it is further recommended that the acquisition of a Stokes-type basket stretcher or scoop stretcher ${ }^{16}$ would be preferable for field-of-play extrication.

The multitude of stadiums internationally make the provision of oxygen cylinders an optional rather than a mandatory item due to the logistical difficulties of having stored oxygen routinely available on scene during training or competition matches. This necessitated including FMEB items that were not compressed gas driven or dependent. Hence the inclusion of a pressurised metered dose inhaler and volumetric spacer rather than a nebuliser system for acute asthma.

Box 1 Emergency medical conditions on the football

field

- Acute anaphylaxis

- Acute asthma

- Acute chest pain

- Dehydration

- Dental injuries

- Dislocations

- Fractures

- Grand mal seizures

- Head injury

- Heat-related emergencies

- Hypoglycaemia

- Spinal injury

- Sudden cardiac arrest

Table 1 Contents of the FIFA emergency medical bag

\begin{tabular}{|c|c|c|}
\hline \multicolumn{3}{|l|}{ Personal protection } \\
\hline Pair of latex/nitrile gloves & $\begin{array}{l}\text { Small/medium/large-as } \\
\text { appropriate }\end{array}$ & 2 \\
\hline Plastic goggles & One size & 1 \\
\hline Antiseptic hand disinfectant & $100 \mathrm{~mL}$ & 1 \\
\hline Rescue scissors shears & Stainless steel & 1 \\
\hline \multicolumn{3}{|l|}{ Airway management } \\
\hline Guedel oropharyngeal tube & Sizes 3 and 4 & 1 each \\
\hline Nasopharyngeal airway & 6 and $7 \mathrm{~mm}$ & 2 each \\
\hline Laryngeal mask airway (LMA) & Sizes 3 and 4 & 1 each \\
\hline Water-based lubricating Jelly & $50 \mathrm{~mL}$ tube & 1 \\
\hline Magill's forceps & Size-adult & 1 \\
\hline Suction hand held & Manual & 1 \\
\hline Suction catheter & Yankuer & 2 \\
\hline Suction catheter & Flexible & 2 \\
\hline Spencer Wells artery forceps & Straight/stainless steel & 2 \\
\hline Scalpel handle and blade & Size 15, disposable & 2 \\
\hline \multicolumn{3}{|l|}{ Breathing } \\
\hline $\begin{array}{l}\text { Bag valve manual resuscitator } \\
\text { (self-inflating) }\end{array}$ & $\begin{array}{l}\text { Without adult-pop off } \\
\text { valve }\end{array}$ & 1 \\
\hline Bag valve face mask & Sizes 3,4 and 5 & 1 each \\
\hline Volumetric spacer device & Size-adult & 1 \\
\hline Pulse oximeter & With batteries & 1 \\
\hline \multicolumn{3}{|l|}{ Circulation } \\
\hline Automated external defibrillator & AED & 1 \\
\hline AED pads & Adult & 1 \\
\hline Shaving type razor & Disposable & 2 \\
\hline Towelling & Polyesterlcotton & 1 \\
\hline Stethoscope & Dual head & 1 \\
\hline Sphygmomanometer & Aneroid clip on & 1 \\
\hline Venous tourniquet—quick release & Size-adult & 1 \\
\hline Hazard sharps & $500 \mathrm{~mL}$ container & 1 \\
\hline $\begin{array}{l}\text { Antiseptic type appropriate swab, } \\
\text { for example, chlorhexidine }\end{array}$ & Sachets & 25 \\
\hline IV cannula & $14,16,18$ and $20 \mathrm{G}$ & 3 each \\
\hline Sterile IV retaining dressing & $7.5 \times 8.5 \mathrm{~cm}$ packet & 4 \\
\hline IV fluid administration set & $15 \mathrm{drop} / \mathrm{mL}$ & 2 \\
\hline Sterile disposable syringe & $\begin{array}{l}\text { Size } 2 \mathrm{~mL}, 5 \mathrm{~mL}, 10 \mathrm{~mL} \text {, } \\
20 \mathrm{~mL}\end{array}$ & 4 each \\
\hline Hypodermic needle & Size $21 \mathrm{~g} \times 40 \mathrm{~mm}$ & 6 \\
\hline Hypodermic needle & Size $18 \mathrm{~g} \times 40 \mathrm{~mm}$ & 6 \\
\hline Lactated Ringer's solution & $500 \mathrm{mLs}$ & 2 \\
\hline Sterile saline & $20 \mathrm{~mL}$ & 5 \\
\hline Arterial tourniquet & Size-adult & 1 \\
\hline Clinical waste bag & $20 \times 25 \mathrm{~cm}$ with seal & 3 \\
\hline \multicolumn{3}{|l|}{ Dressings } \\
\hline Gauze swabs $10 \mathrm{~cm} \times 10 \mathrm{~cm}$ & packet of 5 & 10 \\
\hline Crepe bandage & 100,75 and $50 \mathrm{~mm}$ & 2 each \\
\hline Trauma wound dressing & 100,75 and $50 \mathrm{~mm}$ & 4 each \\
\hline Transpore hypoallergenic tape & $25 \mathrm{~cm}$ & 2 \\
\hline Sterile burn dressing & $100 \mathrm{~mm} \times 100 \mathrm{~mm}$ & 5 \\
\hline Plasters & Assortment of sizes & 1 box \\
\hline Non-suture skin closures & Singles & 5 \\
\hline Adhesive bandage & $25 \mathrm{~mm}$ & 2 \\
\hline Celox gauze dressing & & 1 \\
\hline Protective eye shield & & 1 \\
\hline \multicolumn{3}{|l|}{ Evacuation } \\
\hline Long trauma board & Plasticised & 1 \\
\hline Board immobilisation straps & $\begin{array}{l}6 \times \text { straps/spider type or } \\
\text { equivalent }\end{array}$ & $1 \times$ set \\
\hline Calico triangular bandage & Single wrapped & 6 \\
\hline
\end{tabular}


Table 1 Continued

\begin{tabular}{|c|c|c|}
\hline Lower limb metal traction splint & $\begin{array}{l}\text { Kendrick traction splint } \\
\text { or equivalent }\end{array}$ & 1 \\
\hline $\begin{array}{l}\text { Rescue type blankets to prevent or } \\
\text { treat hypothermia postinjury }\end{array}$ & Aluminium or equivalent & 4 \\
\hline \multicolumn{3}{|l|}{ Fractures } \\
\hline SAM splint orange/blue & $91.5 \mathrm{~cm} \times 11.5 \mathrm{~cm}$ & 2 \\
\hline Ambu head wedge cervical & Single & 2 \\
\hline \multicolumn{3}{|l|}{ General } \\
\hline Sealable plastic bag & Small, medium and large & 2 each \\
\hline Glucometer & With batteries & 1 \\
\hline Glucometer test strips & Singles & Pack of 10 \\
\hline Lancets & Singles & Pack of 10 \\
\hline Black marker + black ink pen & & 1 \\
\hline Thermometer & Non-mercury & 1 \\
\hline Penlight & & 1 \\
\hline Prescription pad/referral letter & & 1 \\
\hline $\begin{array}{l}\text { Team/venue emergency protocol } \\
\text { and contacts }\end{array}$ & & 1 \\
\hline Inventory and checklist & & 1 \\
\hline \multicolumn{3}{|l|}{ Medications } \\
\hline Epinephrine 1 in 1000 injection & $1 \mathrm{mg}$ per $\mathrm{mL}$ & 10 \\
\hline Aspirin tablets & $300 \mathrm{mg}$ dissolvable & 10 \\
\hline Atropine & $0.5 \mathrm{mg}$ in $1 \mathrm{~mL}$ & 5 \\
\hline Chlopheniramine injection & $10 \mathrm{mg}$ in $1 \mathrm{~mL}$ & 5 \\
\hline Cyclisine injection & $50 \mathrm{mg}$ in $1 \mathrm{~mL}$ & 5 \\
\hline Glucose gel & $25 \mathrm{~g}$ sachet & 3 \\
\hline Glyceryl trinitrate tablets/spray & $300 \mu \mathrm{g}$ sublingual & I unit \\
\hline Midazolam & $15 \mathrm{mg}$ in $3 \mathrm{~mL}$ & 3 \\
\hline $\begin{array}{l}\text { Salbutamol pMDI (pressurized } \\
\text { Metered Dose Inhaler) }\end{array}$ & $200 \mu \mathrm{g} /$ dose & 1 \\
\hline $\begin{array}{l}\text { Tramadol injection (or equivalent } \\
\text { analgesic) }\end{array}$ & $50 \mathrm{mg}$ in $2 \mathrm{~mL}$ & 4 \\
\hline Oral rehydration solution sachets & & 10 \\
\hline
\end{tabular}

The contents of the FMEB (figures 1 and 2) have been selected mainly in a generic format such that all of the items can be replaced if used in an emergency or expire using the equivalent locally available stocked items. Additionally the outer compartment of the FMEB has transparent plastic and houses the AED, making the AED presence immediately visible from a distance and easily checked for functionality on a regular basis without having to open the compartment (figures 1 and 2).

\section{Prevention and management of sudden cardiac arrest}

As the leading cause of death in sport, SCA warrants specific considerations in the implementation of a comprehensive programme to respond to life-threatening medical emergencies on the field-of-play. F-MARC has therefore outlined the FIFA 11 Steps for the prevention of sudden cardiac death in football (box 2).

\section{Prevention (steps 1-3)}

Preparticipation screening is universally supported to identify athletes with pre-existing conditions that place them at risk of catastrophic injury or sudden death. The Precompetition Medical Assessment (PCMA) as recommended by FIFA ${ }^{17-19}$ involves at least a focused player medical history (PMH), family medical history $(\mathrm{FMH})$ and cardiac specific physical medical examination. A resting 12-lead ECG should be undertaken as

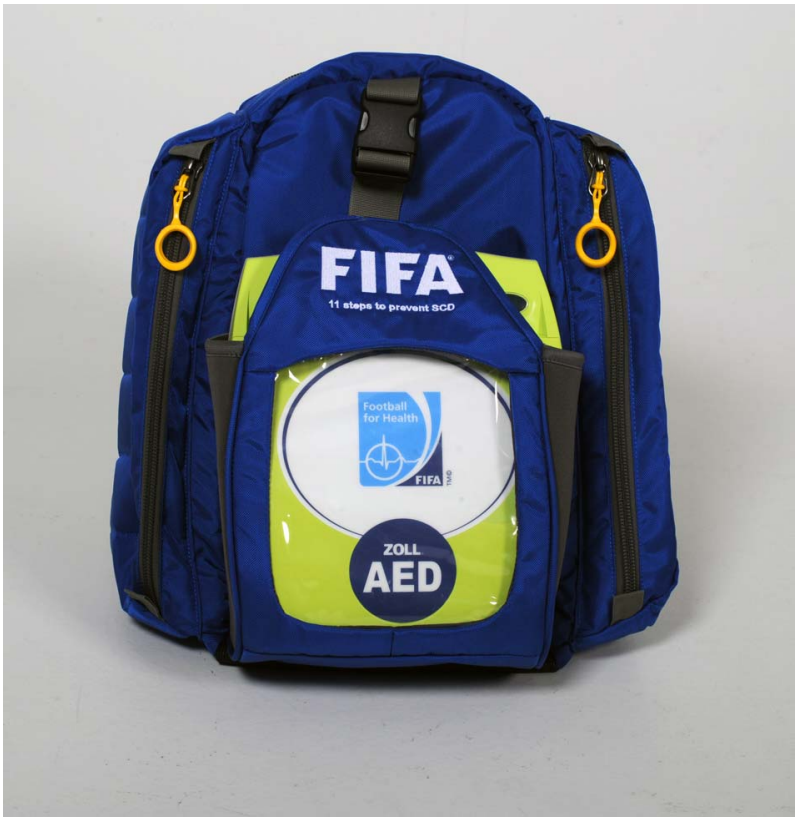

Figure 1 FIFA medical emergency bag.

part of the PCMA on all players at the beginning of their playing career and then once every year. ${ }^{20}{ }^{21}$ Echocardiography should be undertaken by an experienced cardiologist when abnormal results are found on the above history, examination and/or ECG, and should be considered at least once in a player's early career ${ }^{22}$ to better detect structural disorders not routinely identified by ECG. An exercise test should be considered in athletes older than 35 years of age and when otherwise indicated.

\section{Planning + protocol (step 4)}

Appropriate, annual CPR and AED training should be undertaken for all team staff and referees to ensure these life-saving skills are up to date. ${ }^{23}$ The FMEB or equivalent should be acquired by, or available to, each team for use, if and when medical emergencies occur, as part of an approved emergency medical plan (EMP). ${ }^{24}$ All on-duty medical personnel must be familiar and have checked the contents of the FMEB before the game. An approved football team and football stadium EMP should be established that defines what steps are taken, by which football staff, using which equipment, during a medical emergency. The EMP should be part of initial training and revised/practiced at least annually. Prior to each game, roles and responsibilities for each aspect of the EMP should be allotted to medical team members. ${ }^{25}$ The field-of-play medical team is encouraged to review and/or practice retrieval of the AED and other emergency equipment before each match with on-duty personnel present and equipment positioned and accessible.

The official on-duty field-of-play medical team should be comprised of members who are adequately trained, experienced and have appropriate qualifications in football emergency medicine that is practiced on the field and within the stadium. An on-site attending ambulance must be available on location within the football stadium at a designated time and location, staffed with appropriately trained crew and operational with comprehensive medical equipment. Preferably, the ambulance should be located in a position which enables it to enter the field, if and when required, as part of the EMP. 


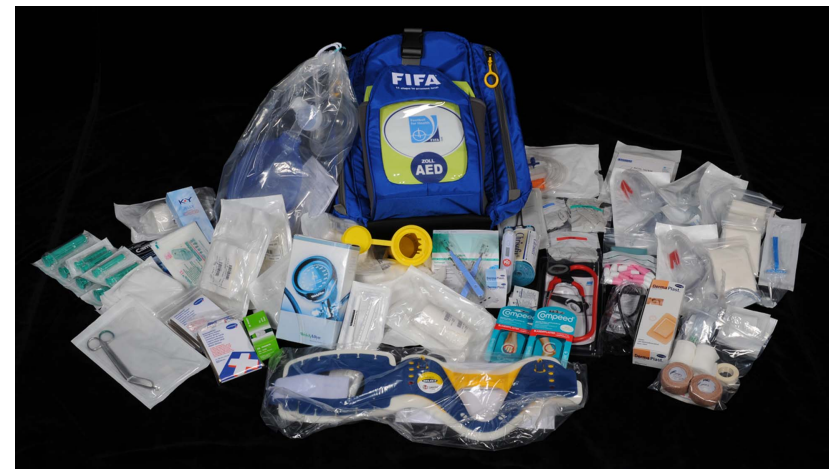

Figure 2 Contents of the FIFA medical emergency bag.

Play the game + pregame timeout (steps $5-7$ )

Before starting of all training sessions and all games, a FMEB with AED must be in position at the field-side. In addition, before starting of all games, an on-duty medical team and an adequately staffed and equipped ambulance must be positioned at the field-side.

\section{Box 2 The FIFA 11 Steps to prevent sudden cardiac} death in football

\section{Prevention}

1. PCMA - player medical history (PMH), family history and physical examination

2. ECG-12-lead, resting, supine; initially + every year

3. Echocardiography - where necessary and at least once in the early career, exercise test where necessary and in athletes $>35$ years old

Planning + protocol

4. Training and equipment

A. CPR + AED training yearly for team staff and referees undertaken

B. FIFA medical emergency bag available and checked

C. Emergency medical plan-roles and responsibilities allotted; on field response practiced and rehearsed at least once annually

D. Field-of-play medical team qualifications + logistics confirmed

E. Ambulance location and logistics confirmed

Play the game + pre-game timeout

5. FIFA medical emergency bag with AED in position and checked

6. Field-of-play medical team in position (games)

7. Ambulance, fully functional, in position (games)

Performance of the emergency medical plan

8. Immediate recognition of collapsed player

A. Assume SCA if collapsed and unresponsive

B. Seizure activity and/or agonal respirations-SCA

9. Activation of emergency medical plan

10. Early CPR and AED application

A. Start chest compressions

B. Retrieve, apply and use AED as soon as possible

11. Early planned transition to advanced life support

$A E D$, automated external defibrillator; CPR, cardiopulmonary resuscitation; PCMA, precompetition medical assessment; SCA, sudden cardiac arrest.
Performance of the emergency medical plan (steps 8-11)

Immediate recognition of any collapsed player is the initial primary responsibility of the field-of-play medical team. Any football player who collapses and is unresponsive, particularly if it occurs without contact with another player, is to be regarded as a SCA until proved otherwise. ${ }^{26}$ Any football player who collapses and displays seizure-like activity and/or agonal respirations should be regarded as a SCA. ${ }^{23}$ For any suspected SCA, the EMP must be activated immediately followed by prompt CPR and retrieval, application and use of an AED as soon as possible. Chest compressions should be started immediately and continued until the AED has been brought to the collapsed player and can be applied to analyse the cardiac rhythm. ${ }^{26}$ After adequate chest compressions and AED use has been undertaken, the player must be transitioned to advanced life support by transferring the player to the ambulance inside the stadium, preferably by summoning the ambulance onto the field to the player's side. If this cannot be done, the player must be safely and quickly transferred to the ambulance with continuous chest compressions and repeat use of an AED if necessary. Effective chest compressions and AED use must also be maintained during ambulance transportation as indicated. ${ }^{27} 28$

If the game is resumed following an on-field rescue, medical personnel are reminded to return to the field-of-play to continue their duties. The EMP should include provisions for a 'return to normal' situation similar to a pregame timeout including the anticipation of back-up ambulance transport and restoration of used medical equipment.

\section{CONCLUSION}

The FMEB and FIFA 11 Steps to prevent SCD are part of a comprehensive preventative programme to appropriately manage $\mathrm{SCA}^{14}{ }^{15}$ and other acute life threatening or serious field-of-play medical emergencies. Both the FMEB and FIFA 11 Steps to prevent SCD are part of a broader preventive philosophy promoting player safety and football as a health enhancing leisure activity. Education of the medical and paramedical staff, coaches, physiotherapists, referees and fitness trainers is an essential adjunct to this process. The FMEB is the proposed medical equipment standard to be provided at every football field and to be used when medical emergencies arise. The complete FMEB was presented to the FIFA Congress 2013 and delivered to all FIFA MAs in June 2013.

Acknowledgements The authors and F-MARC would like to thank FIFA for making the effort and financial commitment to support this unique action. Special appreciation also belongs to the technical staff of the Schulthess Clinic, Zurich for organising and packing the contents of the FMEB, as well as coordinating the logistics for FIFA to distribute the bags around the world.

Contributors All authors were involved in the development and review of the manuscript.

Funding FIFA Medical Assessment and Research Center (F-MARC).

Competing interests None.

Provenance and peer review Commissioned; internally peer reviewed.

\section{REFERENCES}

1 Manning MR, Levy RS. Soccer. Phys Med Rehabil Clin N Am 2006;17:677-95.

2 Kibler WB. Injuries in adolescent and preadolescent soccer players. Med Sci Sports Exerc 1993:25:1330-2.

3 Hanson J, Carlin B. Sports prehospital-immediate care and spinal injury. Br J Sport Med 2012:46:1097-101.

4 Rutherford DS, Niedfeldt MW, Young CC. Medical coverage of high school football in Wisconsin in 1997. Clin J Sport Med 1999:9:209-15.

5 Verral GM, Brukner PD, Seward HG. Doctor on the sidelines. MJA 2006;184:244-8

6 Lohrer $\mathrm{H}$, Malliaropoulos N. On-field sports medicine emergencies: what's new! $\mathrm{Br} J$ Sport Med 2012:46:1089-90. 
7 Kramer EB, Dvorak J, Kloeck WGJ. Review of the management of sudden cardiac arrest on the football field. Br J Sport Med 2010;44:540-5.

8 Drezner JA, Rao AL, Heistand J, et al. Effectiveness of emergency response planning for sudden cardiac arrest in United States high schools with automated external defibrillators. Circulation 2009:120:518-25.

9 Menzies D, Brenna I. EMS equipment. In: Tintinalli J, Cameron P, Holliman CJ. eds. EMS: a practical global guidebook. Shelton, CT: People's Medical Publishing House, 2010:253-84.

10 Rehberg RS. Preparing for sport emergencies. In: Sports emergency care: a team approach. NJ: SLACK Inc, 2007:9-22.

11 Dvorak J, Junge A. Soccer injuries, a review of incidence and prevention. Sports Med 2004:34:929-38.

12 Constantinou D, Kramer EB, Motaung S. In: Dvorak J, Grimm K. eds. FIFA football emergency medicine manual. Zurich: Federation Internationale de Football Association (FIFA), 2010:1.1-4.4.

13 Cunningham A. An audit of first aid qualifications and knowledge among team officials in two English youth football leagues: a preliminary study. $\mathrm{Br}$ I Sports Med 2002;36:295-300.

14 Hanson JR, Carlin B. Sports prehospital-immediate care and spinal injury. Br J Sports Med 2012;46:1097-101.

15 Del Rossi G, Rechtine GR, Conrad BP, et al. Are scoop stretchers suitable for use on spine-injured patients? Am J Emerg Med 2010;28:751-6.

16 Corrado D, Pelliccia A, Bjornstad HH, et al. Cardiovascular pre-participation screening of young competitive athletes for prevention of sudden death. Eur Heart $J$ 2005:26:516-24.

17 Dvorak J, Grimm K, Schmied C, et al. Development and implementation of a standardized pre-competition medical assessment of international elite football players -2006 FIFA World Cup Germany. Clin J Sport Med 2009;19:316-21.
18 FIFA Pre-Competition Medical Assessment (PCMA). http://www.fifa.com/mm/ document/afdeveloping/medical/01/07/26/86/fifapcmaform.pdf (accessed 3 Jan 2013).

19 Corrado D, Pelliccia A, Heidbuchel $\mathrm{H}$, et al. Recommendations for interpretation of 12-lead electrocardiogram in the athlete. Eur Heart J 2010;31:243-59.

20 Drezner JA, Ackerman MJ, Anderson J, et al. Electrocardiographic interpretation in athletes: the 'Seattle criteria'. Br J Sports Med 2013;47:122-4.

21 Keller DI, Bizzini M, Feddermann N, et al. FIFA Women's World Cup 2011: pre-competition medical assessment of female referees and assistant referees. $\mathrm{Br} \mathrm{J}$ Sports Med 2013:47:179-81.

22 Kramer EB. Football emergency medicine. CME 2010;28:208-12.

23 Kramer EB, Botha $\mathrm{M}$, Drezner J, et al. Practical management of sudden cardiac arrest on the football field. Br J Sports Med 2012;46:1094-6.

24 Courson RW. Preventing sudden death on the athletic field: the emergency action plan. Curr Sport Med Rep 2007;6:93-100.

25 Drezner JA, Courson RW, Roberts WO, et al. Inter-association task force recommendations on emergency preparedness and management of sudden cardiac arrest in high school and college athletic programs: a consensus statement. Heart Rhythm 2007:4:549-65.

26 Kreimeier U, Dirks B, Arntz R, et al. Chest compression without ventilation during basic life support? Confirmation of the validity of the European Resuscitation Council (ERC) guidelines 2005. Anaesthesia 2008;57:812-16.

27 Ferguson E. 78 Minutes in the life (and near death) of Fabrice Muamba. http:/ www.guardian.co.uk/football/2012/mar/25/muamba-collapse-minute-by-minute (accessed 3 Jan 2013).

28 n.a. Fabrice Muamba: Doctors re-live race to save Bolton midfielder.. Available at: http://www.bbc.co.uk/sport/0/football/17469449 (accessed 3 Jan 2013). 